A natural solution to increase sample size is to incorporate data from multiple sources, traditionally done through centralization. Unfortunately, this approach presents disadvantages concerning privacy protection. Alternatively, models can be trained in one source and tested in another setting, sharing only the model coefficients (figure 1). This approach does not disclose any privacy-sensitive information. Additionally, external validation provides stronger evidence on the generalizability of the obtained statistical model.

In this study, we used the alternative approach to increase sample size and identify risk factors for postoperative lymph node metastases ( $\mathrm{pN} 1)$ in early-stage cervical cancer.

Methodology We conducted an international observational cohort study using data from the Danish Gynaecologic Cancer Database (DGCD) and the Netherlands Cancer Registry (NCR). We included women diagnosed with early-stage cervical squamous cell carcinoma, adenocarcinoma or adenosquamous carcinoma between 2005-2017, who were treated with radical surgery.

We generated two logistic regression models to identify risk factors for pN1. Model A was trained on NCR data and tested with DGCD data, while model B was obtained the

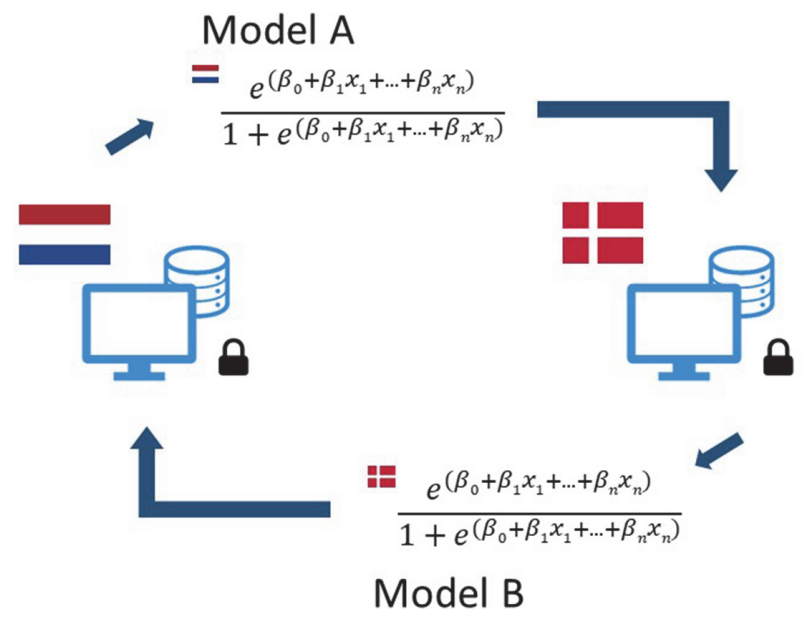

Abstract 1180 Figure 1 A privacy-preserving alternative to small sample analysis. Model A is trained on data from the NCR and tested on DGCD data. Model B was obtained the other way around. Only model coefficients need to be shared and, thereby, no privacy-sensitive information is disclosed
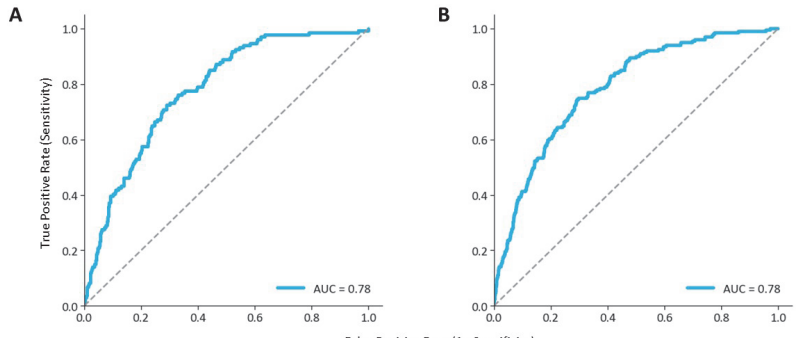

Abstract 1180 Figure 2 Areas under the curve for two models identififying risk factors for postoperative lymph node metastases in earl-stage cervical cancer. 2A: Model A trained with NCR data, tested on DGCD data; 2B: Model B trained with DGCD data, tested on NCR data. other way around. The performance of each model was assessed using areas under the curve (AUCs).

Result(s)* A total of 2735 patients were included in the analyses.

In model A - trained on data from 1476 Dutch patients and tested with data from 1259 Danish patients - tumour size (odds ratio 1.62; 95\% confidence interval [1.37-1.91]), depth of invasion (1.30 [1.12-1.53]) and LVSI (5.13 [3.50-7.52]) were identified as risk factors for $\mathrm{pN} 1$, and body mass index as protective factor $(0.80$ [0.67-0.95].

In model B, trained on Danish data $(n=1259)$ and tested with Dutch data $(n=1476)$, tumour size (1.48 [1.23-1.79]), depth of invasion (1.27 [1.04-1.54]) and LVSI (3.85 [2.535.86]) were associated with increased risk of $\mathrm{pN} 1$.

External validation of model $\mathrm{A}$ and $\mathrm{B}$ both showed AUCs of 0.78 (figure 2).

Conclusion* Tumour size, depth of invasion and LVSI were identified as risk factors for $\mathrm{pN} 1$ in both models.

Separate models, containing either Danish or Dutch data have shown to be valid by privacy-preserving external validation.

\section{RESECTION OF THE UPPER PARACERVICAL LYMPHOVASCULAR TISSUE SHOULD BE A PART OF A PELVIC SENTINEL LYMPH NODE ALGORITHM IN EARLY STAGE CERVICAL CANCER}

O Lührs*, L Ekdahl, B Geppert, C Lönnerfors, J Persson. Lund University Faculty of Medicine, Department of Obstetrics and Gynecology, Lund, Sweden

\subsection{6/ijgc-2021-ESG0.643}

Introduction/Background* To investigate the prevalence of lymph nodes and lymph node metastases (LNMs) in the upper paracervical lymphovascular tissue (UPLT) in early stage cervical cancer.

Methodology In this prospective study consecutive women with stage IA1-IB1 cervical cancer underwent a pelvic lymphadenectomy including identification of sentinel nodes (SLNs) as part of a nodal staging procedure in conjunction with a robotic radical hysterectomy $(\mathrm{RRH})$ or robotic radical trachelectomy (RRT). Indocyanine green (ICG) was used as tracer. The UPLT was separately removed and defined as "SLN-parametrium" and, as all SLN tissue, subjected to ultrastaging and immunohistochemistry. Primary endpoint was prevalence of lymph nodes and metastatic lymph nodes in the UPLT. Secondary endpoints were complications associated with removal of the UPLT.

Result(s)* One hundred and forty-five women were analysed. Nineteen (13.1\%) had pelvic LNMs, all identified by at least one metastatic SLN. In 76 women (52.4\%) at least one UPLT lymph node was identified. Metastatic UPLT lymph nodes were identified in six women of which in three women $(2.1 \%$ of all women and $15.8 \%$ of node positive women) without lateral pelvic LNMs. Thirteen women had lateral pelvic SLN LNMs with either no $(n=5)$ or benign $(n=8)$ UPLT lymph nodes. No intraoperative complications occurred due to the removal of the UPLT.

Conclusion* Removal of the UPLT should be an integral part of the SLN concept in early stage cervical cancer. 